\title{
Human papillomavirus genotyping in low-grade squamous intraepithelial lesions
}

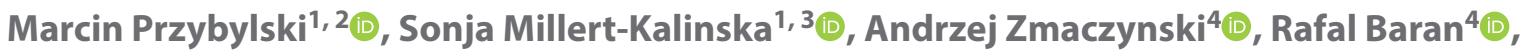 \\ Lucja Zaborowska ${ }^{4}$, Robert Jach ${ }^{4}$, , Dominik Pruski ${ }^{1}, 5(0)$ \\ ${ }^{1}$ Department of Obstetrics and Gynecology, District Public Hospital in Poznan, Poland \\ ${ }^{2}$ Marcin Przybylski M.D. Gynecology Specialised Medical Practice Poznan, Poland \\ ${ }^{3}$ Poznan University of Medical Sciences, Poland \\ ${ }^{4}$ Department of Gynecology and Obstetrics, Jagiellonian University Collegium Medicum, Cracow, Poland \\ ${ }^{5}$ Dominik Pruski M.D. Gynecology Specialised Medical Practice Poznan, Poland
}

\begin{abstract}
Objectives: Human papillomavirus infection is one of the most common sexually transmitted infections. Histological LSIL in $70-80 \%$ of cases will regress spontaneously, while a subset is associated with residual risk for a future precancerous lesion. This study evaluates the performance of HPV genotypes for LSIL preceded by normal or mildly abnormal Pap smear.

Material and methods: We provide a prospective observational cohort study. We obtained material from 428 women registered to Specialist Medical Practice and Provincial Hospital in Poznan in 2018-2021. In the current study, we analyze results from the first 112 inclusions with the diagnosis of LSIL from a cervical biopsy.

The probe for the molecular test was collected with a combi brush and passed to the independent, standardized laboratory. HPV detection was done using PCR followed by DNA enzyme immunoassay and genotyping with a reverse hybridization line probe assay. Sequence analysis was performed to characterize HPV — positive samples with unknown HPV genotypes. The molecular test detected DNA of $41 \mathrm{HPV}$ genotypes. We performed statistical analyzes using the STATISTICA package 13.3.

Results: We found that $77.7 \%$ of patients received HPV-positive test results. The most frequent HPV genotype was 16 , which was assumed for $22.3 \%$. We detected that following HPV types are next most common: HPV 56 (11.6\%), HPV $52(8.9 \%)$, HPV 31 (8.0\%) and HPV 51 (8.0\%). Among HPV 16-negative women, the vast majority are those living in the town $(p=0.048)$. Moreover, thyroid diseases were the most common comorbidities.

Conclusions: To our knowledge, this study is the most extensive assessment of HPV genotypes in LSIL diagnoses in Poland.
\end{abstract} Key words: HPV genotyping; LSIL; low-grade lesion; cervix biopsy

Ginekologia Polska 2022; 93, 1: 18-23

\section{INTRODUCTION}

Human papillomavirus (HPV) infection is a common sexually transmitted disease. A vast majority of the infections resolve within one or two years; however, if not controlled immunologically or by screening, some genotypes may lead to persistent infection resulting in cervical cancer. The carcinogenicity of these HPV types results primarily from the oncoproteins E6 and E7, which impair growth regulatory pathways. It is still unclear which precancerous lesions progress, and which do not [1,2]. Infections with oncogenic HPV genotypes can cause cancer in both women and men. However, cervical tissue is more prone to HPV-dependent cancer development. Thus, human papillomaviruses cause over eight times more cancers in women than in men [3,4].

Cervical cancer remains the fourth most frequent cancer in women worldwide, causing about 275,000 deaths annually $[5,6]$. Moreover, depending on the screening and treatment programs proposed by different countries, the incidence of cervical cancer varies by geographic region. In most countries, prevention programs are based on typical Pap-smear. In 2003, the American College of Obstetricians and Gynecologists first proposed the HR HPV DNA test as screening. Since then, other international societies have 
started to indicate greater effectiveness of this method in middle-aged patients compared to classical Pap-smear [7,8].

Lesions classified as low grade squamous intraepithelial lesions (LSIL) show some heterogeneity. The microscopic image is usually more viral than in inconspicuous infections, and lesions are characterized by cell proliferation through the lower layers of infected cervical tissue.

Randomized trials and systematic reviews show that, compared with repeated Pap-smear, hrHPV testing has higher sensitivity and similar specificity in identifying underlying or incipient cervical precancer in women with ASC-US $[9,10]$. Accordingly, triage by hrHPV testing has become standard practice [7, 11, 12]. Low-grade squamous intraepithelial lesions are associated with a risk for precancer like that among hrHPV-positive women with ASC-US [13]. Because most women with LSIL test positive for hrHPV [14], triage by hrHPV testing is inefficient [15]. The widespread practice of referring all women with hrHPV infection and ASC-US or LSIL to colposcopy carries a considerable burden and cost. Because HPV types 16 and 18 cause about $70 \%$ of cervical cancer cases [12], genotyping for these types have been proposed as an additional tool to allow more fine-tuned management.

This paper summarizes the results of HPV DNA genotyping in women diagnosed with LSIL in Poznań, Poland. So far, we do not have reliable data on the contribution of selected oncogenic HPV types in the formation of cervical pathology in the Polish population. To our knowledge, it is the most extensive analysis that has been described in Poland to date. We aim to provide distribution of particular HPV genotypes concerning age groups in women diagnosed with LSIL.

\section{MATERIAL AND METHODS}

We present a prospective observational cohort study conducted in Specialist Medical Practice and Provincial Hospital in Poznan, Poland. Inclusion criteria were: 1) an abnormal cytological test result ( $\geq$ ASC-US), positive HPV test result or abnormal cervix image, 2) 18 years of age or older. The exclusion criteria were: 1) current pregnancy or pregnancy in the previous three months, 2) insufficient material for HPV genotyping.

We collected the data on relevant medical history, number of sexual partners, parity and living status, using a standardized questionnaire from each subject. In the current study, we analyze results from the first 112 inclusions with the diagnosis of LSIL from a cervical biopsy.

We recruited 428 patients registered to Provincial Hospital in Poznan and Specialist Medical Practice between 2018 and 2021 because of either an abnormal Pap-smear result ( $\geq$ ASC-US) and positive HPV test result or abnormal cervix image resulting in the collection of material for histopathological examination. From all women, we dis- tinguished those diagnosed with low-grade squamous intraepithelial lesions.

The follow-up schedule for all women included cytology every six months - close supervision for two years, then return to the routine screening program. If needed, following colposcopy and LEEP conization was performed. Women diagnosed with either negative for intraepithelial lesion (NILM), a negative result for HPV infection, or a typical cervix image did not require extended diagnosis and returned to the regular screening program.

\section{Pap-smear and HPV genotyping}

Parallel to the Pap-smear, we tested those women for the presence of HPV and determined their genotypes. We obtained material with a cervex-brush from the external os of the cervix and vaginal wall. Then, we placed it into a liquid-based medium, ThinPrep PreserCyt Solution. A quality test, identifies high- risk HPV DNA of the following genotypes: $6,11,16,18$, $26,31,33,35,39,40,42,43,44,45,51,52,53,54,55,56,58$, $59,61,62,64,66,67,68,68 \mathrm{a}, 68 \mathrm{~b}, 69,70,71,72,73,81,82$, $83,84,87, \mathrm{CP} 6108,90$ in vitro. A positive test result confirms the presence of DNA from at least one oncogenic HPV virus.

The probe for a molecular test (Linear Array HPV Genotyping-Roche Diagnostics) was collected with a combi brush and passed to an independent standardized laboratory. HPV detection was done using PCR followed by DNA enzyme immunoassay with a reverse hybridization line probe assay. Sequence analysis was performed to characterize HPV-positive samples with unknown HPV genotypes. The molecular test detected DNA of 41 HPV genotypes.

\section{Colposcopy and LEEP-conization}

If needed, following colposcopy and LEEP-conization were performed. A specialist in gynecologic oncology with 10 years of experience examined colposcopy with SmartOPTIC colposcope. The doctor performed an acetic acid test and a Schiller test in each patient. According to Reid's Colposcopic Index, the colposcopic images were evaluated, assessing the color, lesion boundaries and surface, blood vessels, and iodine test. All colposcopic images were archived. We used the International Federation of Cervical Pathology and Colposcopy classification and recommended by the Polish Society of Colposcopy and Cervical Pathophysiology.

Excisions were done with colposcopic guidance after application of acetic acid 5\% and Lugol's iodine. The sizes of the loops were adequate to the size of the lesions. Finally, the curettage of the cervical canal was performed to obtain endocervix material. Twelve to sixteen paraffin blocks were prepared from each cervical specimen, and four to five sections were examined from each block. Histopathological analysis was performed in an independent laboratory by experienced pathologists. 
All patients gave informed consent to participate in the study. The Bioethics Committee approved the study of the Medical Chamber of Wielkopolska on the $17^{\text {th }}$ of March 2021 (95/2021).

\section{Statistical analysis}

We performed calculations using the statistical package Statistica (ver. 13.3) and graphs - using Excel. Statistical hypotheses were verified at the level of significance of 0.05. We performed the Pearson's Chi-square test to analyze the correlation between individual genotypes and age groups. We searched for other correlations between risk factors and the occurrence of individual diagnoses using Pearson's Chi-square or Yates corrected Chi-square tests.

\section{RESULTS}

The mean age of the entire population was 33 . The majority of patients had less than three children, and more than half lived in the town or city with less than 100,000 inhabitants. About one-third of patients had comorbidities. The most frequent were the thyroid diseases, comprising both hypothyroidism and Hashimoto's disease. Thyroid diseases were the most common comorbidities and occurred in 14 patients, although we did not find statistical significance. We also observed cases of fertility issues, polycystic ovaries syndrome and prediabetes. Table 1 presents the descriptive characteristics of the study group.

A total of 87 patients (77.7\%) were positive for HPV DNA. The quantitative and percentage distribution of individual genotypes is presented in Table 2 . Five genotypes were the

\begin{tabular}{|c|c|}
\hline $\mathrm{n}$ & 112 \\
\hline Age [yrs] & 33.4 \\
\hline \multicolumn{2}{|l|}{ Living status } \\
\hline City $>100,000$ inh. & $47(42.0 \%)$ \\
\hline Town or city $<100,000$ inh. & $65(58.0 \%)$ \\
\hline \multicolumn{2}{|l|}{ Parity } \\
\hline 0 & $60(53.6 \%)$ \\
\hline $1-2$ & $45(40.2 \%)$ \\
\hline$\geq 3$ & $7(6.2 \%)$ \\
\hline Comorbidities & $38(33.9 \%)$ \\
\hline Thyroid disease & $14(12.5 \%)$ \\
\hline PCOS/prediabetes & $9(8.0 \%)$ \\
\hline Fertility issues & $7(6.3 \%)$ \\
\hline \multicolumn{2}{|l|}{ HPV status } \\
\hline$(+)$ & $87(77.7 \%)$ \\
\hline$(-)$ & $25(22.3 \%)$ \\
\hline
\end{tabular}

PCOS - polycystic ovarian syndrome; HPV — human papillomavirus most frequent in the study group - $16(22.3 \%), 56(11.6 \%)$, 52 (8.9\%), 31(8.0\%), 51 (8.0\%). They all belong to high-risk oncogenic HPV types. The HPV genotype 16 accounted for $28.7 \%$ of all HPV-positive patients.

In all age groups, the number of HPV-negative patients was lower than those infected with HPV. We analyzed most frequent genotypes - HPV $16(p=0.691), \operatorname{HPV} 31(p=0.201)$, HPV $52(p=0.363)$ and HPV $56(p=0.785)$.

The relationship between the presence of HPV genotype 16 and the living status turned out to be statistically significant ( $p=0.038$ ). Among HPV 16-negative women, the vast majority are those living in the town (63.2\%). Most peo-

Table 2. Distribution of individual HPV genotypes

\begin{tabular}{|c|c|c|c|}
\hline $\begin{array}{l}\text { HPV } \\
\text { genotype }\end{array}$ & Presence $\mathbf{n}$ & $\begin{array}{l}\text { Presence \% } \\
(n=112)\end{array}$ & $\begin{array}{l}\% \text { of genotypes } \\
(n=152)\end{array}$ \\
\hline 16 & 25 & 22.3 & 16.4 \\
\hline 56 & 13 & 11.6 & 8.6 \\
\hline 52 & 10 & 8.9 & 6.6 \\
\hline 31 & 9 & 8.0 & 5.9 \\
\hline 51 & 9 & 8.0 & 5.9 \\
\hline 53 & 8 & 7.1 & 5.3 \\
\hline 54 & 7 & 6.3 & 4.6 \\
\hline 66 & 7 & 6.3 & 4.6 \\
\hline 84 & 7 & 6.3 & 4.6 \\
\hline 18 & 6 & 5.4 & 3.9 \\
\hline 6 & 5 & 4.5 & 3.3 \\
\hline 42 & 5 & 4.5 & 3.3 \\
\hline 33 & 4 & 3.6 & 2.6 \\
\hline 39 & 4 & 3.6 & 2.6 \\
\hline 61 & 4 & 3.6 & 2.6 \\
\hline 89 & 4 & 3.6 & 2.6 \\
\hline 73 & 3 & 2.7 & 2.0 \\
\hline 35 & 2 & 1.8 & 1.3 \\
\hline 40 & 2 & 1.8 & 1.3 \\
\hline 58 & 2 & 1.8 & 1.3 \\
\hline 59 & 2 & 1.8 & 1.3 \\
\hline 67 & 2 & 1.8 & 1.3 \\
\hline 68 & 2 & 1.8 & 1.3 \\
\hline 81 & 2 & 1.8 & 1.3 \\
\hline CP6108 & 2 & 1.8 & 1.3 \\
\hline 7 & 1 & 0.9 & 0.7 \\
\hline 11 & 1 & 0.9 & 0.7 \\
\hline 43 & 1 & 0.9 & 0.7 \\
\hline 45 & 1 & 0.9 & 0.7 \\
\hline 70 & 1 & 0.9 & 0.7 \\
\hline 87 & 1 & 0.9 & 0.7 \\
\hline
\end{tabular}

HPV — human papillomavirus 
ple living in the city were infected with the HPV genotype 16 (60.0\%). We present this correlation in Table 3.

The most frequent HPV genotypes in HPV-positive women below 30 years of age were: 16 (25.0\%), 31(14.3\%) and 56 (14.3\%). The most frequent HPV genotypes in HPV-positive women between 30 and 40 years of age were: 16 (30.6\%), 52 (14.3\%) and 56 (12.2\%). The most frequent HPV genotypes in HPV-positive women above 40 were: 16 (30.0\%) and 56 (30.0\%).

We have found that single HPV infections are more common in women over 30 than infections with multiple HPV types. Only in the youngest group of patients, there was the same frequency of single and multiple infections. In women between 30 and 40, infection with a single virus type occurred in $59.2 \%$ of HPV-positive patients and multiple infections in $40.8 \%$ of patients. $60 \%$ of HPV-positive women were infected with a single HPV type in the oldest age group, and $40.0 \%$ had multiple infections.

The most frequent Pap-smear results in women below 30 years of age were: LSIL and ASC-US. The most frequent Pap-smear results in women above 30 years of age were: LSIL, ASC-US and ASC-H. We have compiled all Pap-smears in Figure 1.

The biopsy in all patients was preceded by colposcopy. We present the results of colposcopy in Figure 2. What

\begin{tabular}{|l|l|l|l|l|l|l|l|l|}
\multicolumn{7}{|c|}{ Table 3. Dependence of HPV 16 on place of residence } & \multirow{2}{*}{ X $^{2}$} & p \\
\hline \multirow{2}{*}{$\begin{array}{l}\text { Living } \\
\text { status }\end{array}$} & \multicolumn{6}{|l|}{ HPV genotype 16 presence } \\
\cline { 2 - 9 } & $\mathbf{n}$ & $\%$ & $\mathbf{n}$ & $\%$ & $\mathbf{n}$ & $\%$ & & \\
\hline City & 32 & 36.8 & 15 & 60.0 & 47 & 42.0 & & \\
\hline Town & 55 & 63.2 & 10 & 40.0 & 65 & 58.0 & 4.30 & 0.038 \\
\hline All & 87 & 100.0 & 25 & 100.0 & 112 & 100.0 & & \\
\hline
\end{tabular}

HPV — human papillomavirus

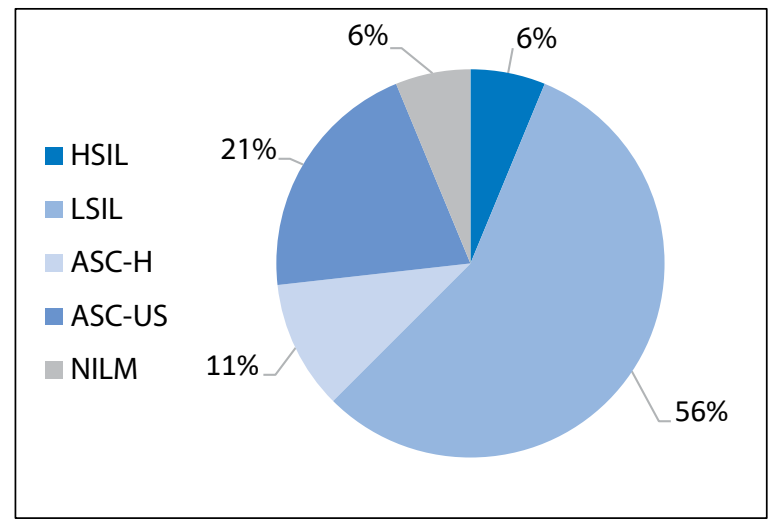

Figure 1. Pap-smear results; HSIL — high-grade squamous intraepithelial lesion; LSIL — low-grade squamous intraepithelial lesion; ASC-H - atypical squamous cells cannot exclude HSIL; ASC-US - atypical squamous cells of undetermined significance; NILM - negative for intraepithelial lesion or malignancy draws attention is the diversity of results according to the Reid Colposcopy Index. As far as follow up is concerned, we performed LEEP-conization in 28 cases. In over half of the patients (15/28), the final diagnosis was milder than one from biopsy- either no pathology was found, or koilocytes were present. Low squamous intraepithelial lesions were detected in one-third of the patients and HSIL changes- in $5 / 28$ of the women. Almost $18 \%$ of cases of the LSIL in biopsy turned out to be HSIL in LEEP-conization. Among the diagnoses of NILM in LEEP-conization, 76.9\% (10/13) of patients were HPV (+). Eighty percent of patients finally diagnosed with LSIL were HPV (+). Among the diagnoses of HSIL in LEEP-conization, all patients were HPV (+). The most common Pap-smear result was LSIL 41.4\% (12/29) and HSIL 27.6\% (8/29). Apart from LEEP-conization, nine patients required another biopsy, of which five had confirmed LSIL lesions, and four showed no pathological changes. Additionally, twenty-two patients, after biopsy, decided to be vaccinated with a 9-valent HPV vaccine. In control, $77.3 \%$ of the women (17/22) were HPV-negative. In contrast, 4/22 post-vaccination patients had recurrent infection with the high-oncogenic HPV genotypes.

\section{DISCUSSION}

The main goal of our study was to determine the distribution of HPV genotypes in patients with LSIL lesions. To our knowledge, it is the most extensive assessment of HPV genotype in LSIL in Poland to date. Additionally, we have not found such a database of one roof patients.

There were 31 HPV genotypes detected in our study group. Our analysis confirmed that HPV genotype 16 was most common. The most frequent HPV genotypes in HPV-positive women below 30 years of age were: 16, 31 and 56, in the group of women between 30 and 40 years of age: 16,52 and 56 and in women above $40: 16$ and 56 .

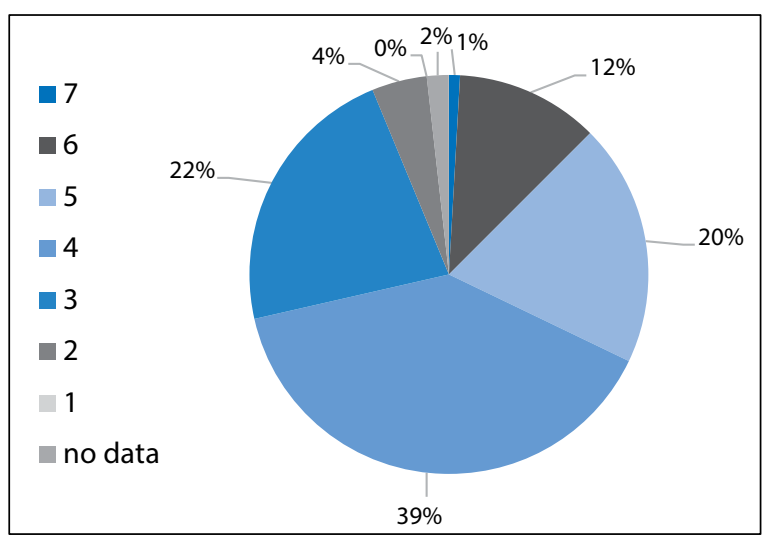

Figure 2. Colposcopy results using Reid Colposcopic index (RCI) 
We have found that single HPV infections are more common in women over 30 than infections with multiple HPV types. Only in the youngest group of patients was there the same frequency of single and multiple infections. In women between 30 and 40, infection with a single virus type occurred in $59.2 \%$ of HPV-positive patients and multiple infections in $40.8 \%$ of patients. $60.0 \%$ of HPV-positive women were infected with a single HPV type in the oldest age group, and $40.0 \%$ had multiple infections.

The discrepancy in the colposcopy results relating to the same biopsy result may indicate that it is worth taking biopsies and not limited to visual assessment. It is a slightly invasive study because HSIL lesions were detected in five patients in the subsequent LEEP conization. Some researchers say that the diagnosis of LSIL associates with a significant increase in the level of stress in patients [16]. The untreated LSIL lesions go into spontaneous remission in the majority of women. Only a fraction of them will contribute to the development of more malignant lesions [17].

A study conducted about a decade ago in the same region of Poland showed that one-third of patients experienced disease regression during the year of follow-up. In $41 \%$ of patients, the LSIL lesion remained at the same level for one year. However, although none of the women developed ICC, a quarter of the study group in the control biopsy progressed to HSIL [18].

High-risk HPV genotypes are closely related to the development of cervical cancer and its precursors [19]. There are different ways of transforming from LSIL lesion to malignancy. Because of that, knowledge about the HPV genotype could be used in separating HPV-positive women at a higher risk of cancer from those that can be observed without intervention over longer intervals. Widespread use of HPV genotyping would improve the efficiency of screening programs while reducing the tendency to over-treatment. [20-22].

HPV as a known carcinogen has led to the development of effective preventive vaccines and sensitive HPV DNA and RNA tests. The analysis of genotypes occurring in patients before and after HPV vaccination may improve their quality in the future [23]. Although screening tests and preventive vaccination programs can significantly reduce the rate of cancer development, their systematic implementation has been a great challenge all over the world for decades [1].

A meta-analysis conducted by Clifford G. et al. [24] on 8308 patients showed that HPV genotype distribution was assessed by geographic region. Almost half of the data (49\%) came from European databases to compare the results of this meta-analysis with our observations. However, Poland did not participate in data transmission to the abovementioned publication, which may constitute a certain limitation. A total of $71.1 \%$ of patients diagnosed with LSIL turned out to be HPV-positive compared to $77.7 \%$ of those observed in our research group. The most common HPV genotype were: HPV16 (present in $26.3 \%$ of all HPV-positive LSILs), HPV 31 (11.5\%), HPV 51 (10.6\%), HPV 53 (10.2\%), HPV 56 (9.5\%), HPV 52 (9.0\%), HPV 18 (8.6\%), HPV 66 (8.6\%), HPV 58 (8.4\%), HPV 6 (8.0\%), HPV 39 (7.6\%), HPV 33 (7.4\%), HPV 59 (6.1\%), HPV 35 (5.7\%), and HPV 45. HPV genotypes occurring less than $5 \%$ were not considered.

The development of a diagnostic and therapeutic strategy in patients with abnormal cytology may in the future depend on the knowledge of the most common HPV genotypes in each region [24-26]. Disseminating this information in different parts of the world may, firstly, provide important epidemiological information. In addition, and more importantly, data collected from large research groups could directly translate into the design of multivalent prophylactic vaccines. Nonetheless, these data on the prevalence and distribution of HPV genotypes in the population may raise the understanding of the HPV molecular epidemiology in Poland.

\section{CONCLUSIONS}

To our knowledge, this study is the most extensive assessment of HPV genotypes in LSIL diagnoses in Poland. Genotyping of human papillomaviruses in the population of women diagnosed with LSIL may help in the future to predict whether LEEP-conization and further invasive diagnostics will be necessary. Our research will make possible to show which viruses are the most common in the Polish population.

\section{Funding}

This research received no external funding.

\section{Institutional review board statement}

The study was conducted according to the guidelines of the Declaration of Helsinki and approved by the Bioethics Committee of the Medical Chamber of Wielkopolska (protocol code 95/2021, date of approval: 17.03.2021).

\section{Informed consent statement}

Informed consent was obtained from all subjects involved in the study.

\section{Data availability statement}

The data presented in this study are available on request from the first and second author. The data are not publicly available due to sensitive information regarding both the health and epidemiological status of the study group.

\section{Conflict of interest}

The authors declare no conflict of interest. 


\section{REFERENCES}

1. Schiffman M, Doorbar J, Wentzensen N, et al. Carcinogenic human papillomavirus infection. Nat Rev Dis Primers. 2016; 2: 16086, doi: 10.1038/nrdp.2016.86, indexed in Pubmed: 27905473.

2. Rodríguez AC, Schiffman M, Herrero R, et al. Proyecto Epidemiológico Guanacaste Group. Rapid clearance of human papillomavirus and implications for clinical focus on persistent infections. J Natl Cancer Inst. 2008; 100(7): 513-517, doi: 10.1093/jnci/djn044, indexed in Pubmed: 18364507.

3. World Health Organisation. INTERNATIONAL AGENCY FOR RESEARCH ON CANCER IARC Monographs on the Evaluation of Carcinogenic Risks to Humans VOLUME 90 Human Papillomaviruses. Human Papillomaviruses. 2007; 90.

4. IARC. Biological agents volume $100 \mathrm{~B}$ A review of human carcinogens: IARC monographs evaluate carcinogenic risks to humans. IARC Monographs. 2012; 100.

5. Dijkstra MG, Snijders PJF, Arbyn M, et al. Cervical cancer screening: on the way to a shift from cytology to full molecular screening. Ann Oncol. 2014; 25(5): 927-935, doi: 10.1093/annonc/mdt538, indexed in Pubmed: 24445150 .

6. Rees I, Jones $\mathrm{D}, \mathrm{Chen} \mathrm{H}$, et al. Interventions to improve the uptake of cervical cancer screening among lower socioeconomic groups: A systematic review. Prev Med. 2018; 111:323-335, doi: 10.1016/j.ypmed.2017.11.019, indexed in Pubmed: 29203349.

7. Saslow D, Solomon D, Lawson HW, et al. American Cancer Society, American Society for Colposcopy and Cervical Pathology, American Society for Clinical Pathology. American Cancer Society, American Society for Colposcopy and Cervical Pathology, and American Society for Clinical Pathology screening guidelines for the prevention and early detection of cervical cancer. Am J Clin Pathol. 2012; 137(4): 516-542, doi: 10.1309/AJCPTGD94EVRSJCG, indexed in Pubmed: 22431528.

8. Karsa Lv, Arbyn M, Vuyst HDe, et al. European guidelines for quality assurance in cervical cancer screening. Summary of the supplements on HPV screening and vaccination. Papillomavirus Research. 2015; 1: 22-31, doi: 10.1016/j.pvr.2015.06.006.

9. Arbyn M, Buntinx F, Van Ranst M, et al. Virologic versus cytologic triage of women with equivocal Pap smears: a meta-analysis of the accuracy to detect high-grade intraepithelial neoplasia. J Natl Cancer Inst. 2004; 96(4): 280-293, doi: 10.1093/jnci/djh037, indexed in Pubmed: 14970277.

10. Arbyn M, Ronco G, Anttila A, et al. Evidence regarding human papillomavirus testing in secondary prevention of cervical cancer. Vaccine. 2012; 30 Suppl 5: F88-F99, doi: 10.1016/j.vaccine.2012.06.095, indexed in Pubmed: 23199969.

11. Money D, Roy M, Scrivener J, et al. Canadian Consensus Guidelines on Human Papillomavirus. Journal of Obstetrics and Gynaecology Canada. 2007; 29(8): S1, doi: 10.1016/s1701-2163(16)32573-7.

12. Arbyn M, Anttila A, Jordan J, et al. European Guidelines for Quality Assurance in Cervical Cancer Screening. Second edition--summary document. Ann Oncol. 2010; 21(3):448-458, doi: 10.1093/annonc/mdp471, indexed in Pubmed: 20176693.

13. ASCUS-LSIL Traige Study (ALTS) Group. A randomized trial on the management of low-grade squamous intraepithelial lesion cytology interpretations. Am J Obstet Gynecol. 2003; 188(6): 1393-1400, doi: 10.1067/mob.2003.462, indexed in Pubmed: 12824968.
14. Zuna RE, Wang SS, Rosenthal DL, et al. ALTS Group. Determinants of human papillomavirus-negative, low-grade squamous intraepithelial lesions in the atypical squamous cells of undetermined significance/low-grade squamous intraepithelial lesions triage study (ALTS). Cancer. 2005; 105(5): 253-262, doi: 10.1002/cncr.21232, indexed in Pubmed: 15991244.

15. Arbyn M, Roelens J, Simoens $C$, et al. Human papillomavirus testing versus repeat cytology for triage of minor cytological cervical lesions. Cochrane Database Syst Rev. 2013(3): CD008054, doi: 10.1002/14651858.CD008054.pub2, indexed in Pubmed: 23543559.

16. Rogstad KE. The psychological impact of abnormal cytology and colposcopy. BJOG. 2002; 109(4): 364-368, doi: 10.1111/j.1471-0528.2002. 99023.x, indexed in Pubmed: 12013155.

17. McCredie MRE, Sharples KJ, Paul C, et al. Natural history of cervical neoplasia and risk of invasive cancer in women with cervical intraepithelia neoplasia 3: a retrospective cohort study. Lancet Oncol. 2008; 9(5): 425-434, doi: 10.1016/S1470-2045(08)70103-7, indexed in Pubmed: 18407790.

18. Pruski D, Fraszczak J, Iwaniec K, et al. Assessment of frequency of regression and progression of mild cervical neoplasia--LGSIL in women with positive high-risk HPV DNA test result. Ginekol Pol. 2012; 83(8): 572-575, indexed in Pubmed: 23342878.

19. Bosch FX, de Sanjosé S. Chapter 1: Human papillomavirus and cervical cancer--burden and assessment of causality. J Natl Cancer Inst Monogr. 2003(31): 3-13, doi: 10.1093/oxfordjournals.jncimonographs.a003479, indexed in Pubmed: 12807939.

20. Schlecht NF, Platt RW, Duarte-Franco E, et al. Human papillomavirus infection and time to progression and regression of cervical intraepithelial neoplasia. J Natl Cancer Inst. 2003; 95(17): 1336-1343, doi: 10.1093/jnci/djg037, indexed in Pubmed: 12953088.

21. Franco EL. Chapter 13: Primary screening of cervical cancer with human papillomavirus tests. J Natl Cancer Inst Monogr. 2003(31): 89-96, doi: 10.1093/oxfordjournals.jncimonographs.a003488, indexed in Pubmed: 12807951.

22. Solomon D. Chapter 14: Role of triage testing in cervical cancer screening. J Natl Cancer Inst Monogr. 2003(31): 97-101, doi: 10.1093/oxfordjournals.jncimonographs.a003489, indexed in Pubmed: 12807952.

23. Galloway DA. Papillomavirus vaccines in clinical trials. Lancet Infect Dis. 2003; 3(8): 469-475, doi: 10.1016/s1473-3099(03)00720-5, indexed in Pubmed: 12901889.

24. Clifford GM, Smith JS, Aguado T, et al. Comparison of HPV type distribution in high-grade cervical lesions and cervical cancer: a meta-analysis. $\mathrm{Br}$ J Cancer. 2003; 89(1): 101-105, doi: 10.1038/sj.bjc.6601024, indexed in Pubmed: 12838308

25. Muñoz N. Human papillomavirus and cancer: the epidemiological evidence. J Clin Virol. 2000; 19(1-2): 1-5, doi: 10.1016/s1386-6532(00)001256, indexed in Pubmed: 11091143

26. Castellsagué $X$, Díaz $M$, de Sanjosé $S$, et al. International Agency for Research on Cancer Multicenter Cervical Cancer Study Group. Worldwide human papillomavirus etiology of cervical adenocarcinoma and its cofactors: implications for screening and prevention. J Natl Cancer Inst. 2006; 98(5): 303-315, doi: 10.1093/jnci/djj067, indexed in Pubmed: 16507827. 